\title{
Novel Ocular and Inner Ear Anomalies in a Patient with Myhre Syndrome
}

\author{
Semra Gürsoy ${ }^{\text {a }}$ Filiz Hazan $^{\text {b }}$ Tülay Öztürk $^{c}$ Halil Ateş $^{d}$ \\ ${ }^{a}$ Department of Pediatric Genetics, Dr. Behcet Uz Children's Hospital, Izmir, Turkey; ${ }^{b}$ Department of Medical \\ Genetics, Dr. Behcet Uz Children's Hospital, Izmir, Turkey; ${ }^{\circ}$ Department of Pediatric Radiology, Dr. Behcet Uz

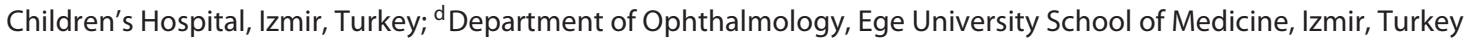

\section{Established Facts}

- Myhre syndrome is a rare autosomal dominant disorder which is caused by gain-of-function mutations in the SMAD4 gene.

- This syndrome is also a multisystem connective tissue disorder which usually affects cardiovascular, respiratory, and gastrointestinal systems. Additionally, the other common features are eye abnormalities (especially strabismus and refractive errors) and early-onset deafness.

\section{Novel Insights}

- We report a 9.6-year-old Turkish patient with molecularly confirmed Myhre syndrome, who had novel eye abnormalities including Axenfield Rieger anomaly and glaucoma in both eyes. Therefore, a regular ophthalmologic evaluation is recommended in these patients.

- Hearing loss is observed in most of the patients, and the underlying etiology of the hearing loss is often unclear or unknown. In our patient, bilateral enlarged vestibular aqueduct was detected by temporal bone $\mathrm{CT}$, and this malformation may be one of the causative factors for hearing loss in this syndrome.

- Temporal bone CT should be performed to evaluate the structural malformations of the inner ear, especially in patients with hearing loss.

\section{Keywords}

Axenfield Rieger anomaly - Enlarged vestibular aqueduct . Glaucoma Myhre syndrome

\section{Abstract \\ Myhre syndrome is a rare autosomal dominant multisystem- ic disorder. Typical features of this disorder include distinc-}

tive facial appearance, deafness, intellectual disability, cardiovascular abnormalities, short stature, brachydactyly, and skeletal anomalies. Gain-of-function mutations in the SMAD4 gene are responsible for this syndrome. Herein, we present a 9.6-year-old Turkish girl with molecularly confirmed Myhre syndrome who had novel findings including bilateral Axenfield Rieger anomaly with secondary glaucoma and bilateral enlarged vestibular aqueducts.

\section{KARGER}

(c) 2019 S. Karger AG, Basel

E-Mail karger@karger.com

www.karger.com $/ \mathrm{msy}$
Semra Gürsoy

Department of Pediatric Genetics

Dr. Behcet Uz Children's Hospital

Sezer Doğan Street, No.11, Izmir 35210 (Turkey)

E-Mail dr.semra@ hotmail.com 
Myhre syndrome (OMIM 139210) is a rare autosomal dominant disorder, which was first described by Myhre et al. [1981]. The patients may have typical facial dysmorphic characteristics including short palpebral fissures, hypoplastic maxilla, midfacial hypoplasia, short philtrum, a narrow mouth, prognathism, small ears, stiff skin, muscular pseudohypertrophy, and difficulty in lifting arms above the shoulders. Skeletal abnormalities including short stature, thickening of the skull bones, platyspondyly, large vertebral pedicles, broad ribs, brachydactyly, and limited joint mobility can also be detected. This syndrome is also a multisystem connective tissue disorder which usually affects the cardiovascular, respiratory, and gastrointestinal system as well as the skin. At least one abnormal eye finding, especially strabismus and refractive errors, was reported in approximately half of the affected patients [Starr et al., 2017]. Other commonly associated features are early-onset deafness of mixed, conductive or sensory type, behavioral disorder, mild to moderate intellectual disability, congenital heart disease, cryptorchidism, and delayed/precocious puberty [Le Goff et al., 2014; Starr et al., 2017].

Myhre syndrome is caused by gain-of-function mutations in the SMAD4 (OMIM 600993) gene which encodes a protein that has an important role in transforming growth factor $\beta$ (TGF- $\beta$ ) and bone morphogenetic proteins signal transduction. In current literature, more than 50 affected individuals with a molecularly confirmed diagnosis have been reported [Starr et al., 2017]. A restricted spectrum of Ile500 mutations (amino acid changes at threonine, valine, and methionine) was found in almost all patients, with the exception of a c.1486C $>\mathrm{T}$ (p.Arg496Cys) change, currently limited to 3 patients [Caputo et al., 2014; Michot et al., 2014]. To date, no clear genotype-phenotype correlations have been defined in affected individuals with either codon abnormality. Herein, we present a 9.6-year-old Turkish girl with molecularly confirmed Myhre syndrome who had novel ocular and inner ear findings.

\section{Case Presentation}

A 9.6-year-old girl was referred to the genetic clinic because of dysmorphic features and mild intellectual disability. She was born to nonconsanguineous Turkish parents by cesarean section after 30 weeks of gestation complicated by preeclampsia and a birth weight of $1,100 \mathrm{gr}(10-50$ th centile). Family history was unremarkable. Physical examination showed a body height of $129 \mathrm{~cm}(10-$ 25th centile), body weight of $33 \mathrm{~kg}$ (50-75th centile), and a head circumference of $52 \mathrm{~cm}$ (50th centile). Facial features included short upslanting palpebral fissures, prominent forehead, short philtrum, bulbous nasal tip, thin lips, small mouth, prognathism, midface hypoplasia, and a high anterior hairline (Fig. 1A). She also had brachydactyly and fifth-finger clinodactyly (Fig. 1B). Thickening of the skin and muscular pseudohypertrophy were detected, but the patient did not have any joint contractures.

Abdominal ultrasonographic examination was unremarkable. Axenfield Rieger anomaly and anterior synechia were detected in both eyes, more prominent in the left eye (Fig. 1D-F). The patient was also under medical treatment for bilateral glaucoma, which was secondary to Axenfield Rieger anomaly. She had monocular esotropia in the left eye. Additionally, cornea, lens, and macula were normal. The pupils were located in the center of the iris; corectopia was not detected (Fig. 1D). Skeletal survey was performed and short middle phalanges, mild broad ribs, mild thickening of the calvarium, and large vertebral pedicles between T11 and L2 were detected (Fig. 1C, G-J). The hearing test revealed a mixed hearing loss (right $35 \mathrm{~dB}$, left $85 \mathrm{~dB}$ ). The girl had already been wearing a hearing aid for 4 years. Temporal bone CT detected bilateral enlarged vestibular aqueducts (EVA) (Fig. 1K). The patient was under anti-hypertensive treatment for the last 4 months and echocardiograhy showed left ventricular hypertrophy.

\section{Materials and Methods}

Genomic DNA from the peripheral blood lymphocytes of all individuals was extracted with QIAamp DNA Blood Mini Kit (Qiagen GMBH, Hilden, Germany) using standard procedures. All coding exons and exon-intron boundaries of SMAD4 were amplified by polymerase chain reaction. The sequences were evaluated using CLC Genomics Workbench 3 sequencing program (Qiagen). The Ensembl database (GRCh38.p12) with ENST00000342988.7 transcript ID of SMAD4 was used to compare the individual's and the reference sequence.

\section{Results}

Routine laboratory tests and immunoglobulin levels were within normal limits. Chromosome analysis confirmed a 46,XX karyotype. Thereafter, SMAD4 gene sequence analysis was performed on the basis of the clinical findings. A heterozygous missense mutation, c.1498A $>\mathrm{G}$ (p.I500V) was detected (Fig. 1L). Neither parent had this gene mutation.

\section{Discussion}

In this report, we describe a patient with Myhre syndrome who had Axenfield Rieger anomaly and glaucoma in both eyes. The patient had bilateral mixed hearing loss, and bilateral enlarged vestibular aqueducts were detected by CT. Myhre syndrome is an increasingly recognized 

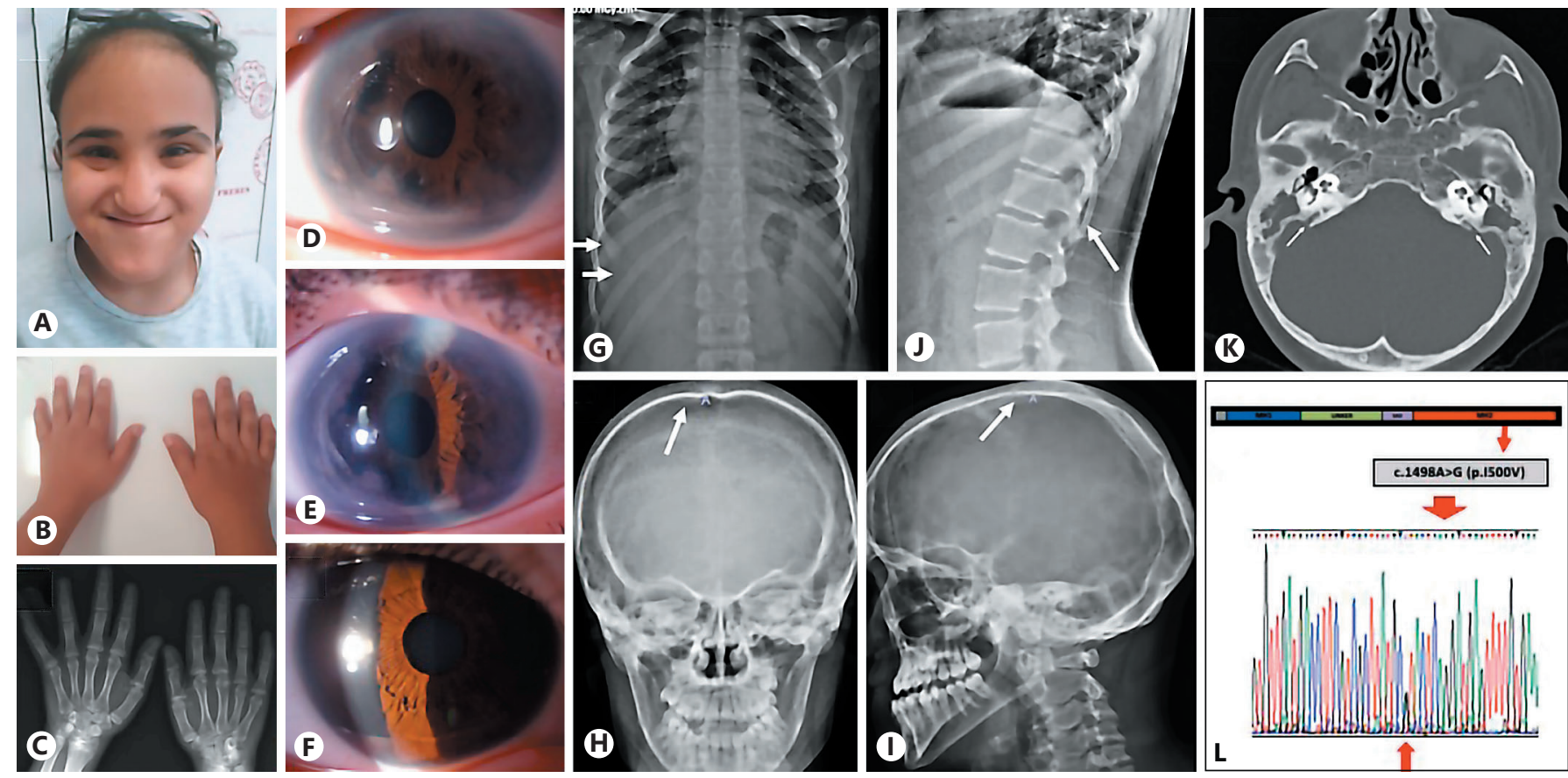

Fig. 1. Dysmorphic features and molecular result of the patient. A Dysmorphic facial features of the patient: short and upslanting palpebral fissures, prominent forehead, short philtrum, bulbous nasal tip, thin lips, small mouth, prognathism, midface hypoplasia, and high anterior hairline. B Photograph of the patient's hands showing brachydactyly and fifth-finger clinodactyly. C X-rays of

disorder which is often diagnosed clinically by typical dysmorphic characteristics. Cardiovascular, respiratory, and gastrointestinal systems may also be affected [Starr et al., 2017].

Myhre syndrome is caused by a heterozygous SMAD4 gain-of-function pathogenic variant. SMAD4 encodes a protein of the SMAD (mothers-against-DPP homolog) family, which is considered as a tumor suppressor. It acts as a comediator within a complex composed of heterodimers of the receptor-regulated SMADs. This complex translocates into the nucleus to induce or repress the expression of TGF- $\beta$ and bone morphogenetic protein target genes, which play an essential role in organogenesis and tissue homeostasis during the developmental process [Starr et al., 2017]. Smad4 is also expressed in both the lens vesicle and presumptive corneal ectoderm, and conditional deletion of Smad4 in the eye surface ectoderm leads to severe abnormality in the anterior segment. Additionally, TGF- $\beta$ signaling can induce epithelial-mesenchymal transitions, which play an important role in the pathogenesis of anterior subcapsular cataract and the hands showing brachydactyly, especially in the middle phalanges. D, E Ocular findings of the left eye. F Photograph of the right eye. G-J X-rays showing mild broad ribs (G), mild thickening of the calvarium $(\mathbf{H}, \mathbf{I})$, and large vertebral pedicles between T11 and L2 (J). K Temporal bone CT showing enlarged vestibular aqueduct. $\mathbf{L}$ Sanger sequence result of the patient.
Table 1. Ocular findings of the patients with molecularly confirmed Myhre syndrome

\begin{tabular}{ll}
\hline Ocular features & $\begin{array}{l}\text { Patients with abnormality/ } \\
\text { patients in the literature, (\%) }\end{array}$ \\
\hline Anterior segment anomalies & $8 / 66(12.1)$ \\
Axenfield Rieger anomaly with glaucoma & $1 / 66(1.5)$ \\
Corectopia & $1 / 66(1.5)$ \\
Cataract & $6 / 66(9)$ \\
Posterior segment anomalies & $6 / 66(9)$ \\
Pseudopapilledema & $3 / 66(4.5)$ \\
Papilledema & $1 / 66(1.5)$ \\
Retinitis pigmentosa and maculopathy & $2 / 66(3)$ \\
Other findings & $31 / 66(46.9)$ \\
Refractive errors & $18 / 66(27.2)$ \\
Strabismus & $13 / 66(19.6)$ \\
\hline
\end{tabular}

Includes 53 cases of the literature reviewed by Lin et al. [2016] and the additional cases by Garavelli et al. [2016], Nomura et al. [2017], Alagia et al. [2018], Erdem et al. [2018], Alape et al. [2019], Artemios et al. [2019], Yu et al. [2019], and the present case. 
Table 2. Congenital malformations of the middle and inner ear and hearing status of mutation-positive patients with Myhre syndrome

\begin{tabular}{lc}
\hline Ear findings & $\begin{array}{c}\text { Patients with abnormality/ } \\
\text { patients in the literature, (\%) }\end{array}$ \\
\hline Congenital malformations or other abnormalities of the middle and inner ear & $6 / 67(8.9)$ \\
Dysplasia of the external semicircular canal, with a thick stapedial footplate fused with the oval window & $1 / 67(1.4)$ \\
Otospongiosis and vestibular schwannoma & $1 / 67(1.4)$ \\
Ossicular fixation & $1 / 67(1.4)$ \\
Chronic inflammatory aspects, thickened tympanic membrane, and mastoid sclerosis & $1 / 67(1.4)$ \\
Small and dysplastic lateral semicircular canals & $1 / 67(1.4)$ \\
Enlarged vestibular aqueduct & $1 / 67(1.4)$ \\
Hearing loss & $52 / 67(77.6)$ \\
\hline
\end{tabular}

Includes Whiteford et al. [2001], Burglen et al. [2003], Kenis et al. [2014], 54 cases of the literature reviewed by Lin et al. [2016] and the additional cases by Garavelli et al. [2016], Nomura et al. [2017], Alagia et al. [2018], Erdem et al. [2018], Alape et al. [2019], Artemios et al. [2019], Yu et al. [2019], and the present case.

posterior capsular opacification. However, the precise role of Smad4 in anterior segment development and the underling mechanism are still unclear [Li et al., 2016]. The 4 pathogenic missense variants at the codons 496 and 500 (p.Arg496Cys, p.Ile500Val, p.Ile500Thr, and p.Ile500Met), which are located in the Mad homology 2 domain, are found in most of the patients with Myhre syndrome [Lin et al., 2016; Starr et al., 2017]. No inactivating deletions or duplications have been reported in the patients. Loss-of-function mutations in the gene may lead to juvenile polyposis syndrome and a spectrum of acquired cardiac diseases [Starr et al., 2017].

Eye abnormalities are present in approximately half of the patients with Myhre syndrome [Starr et al., 2017]. Refractive errors and strabismus are the most common features. Ocular involvement with cataracts, retinitis pigmentosa, maculopathy, papilledema, and pseudopapilledema has been reported in current literature [Garavelli et al., 2016; Erdem et al., 2018]. Alagia et al. [2018] reported a patient with Myhre syndrome who had only corectopia. In our case, the patient had bilateral Axenfield Rieger anomaly with anterior synechia, which has not been reported previously in Myhre syndrome. The patient also had bilateral glaucoma secondary to the Axenfeld Rieger anomaly. Corectopia was not detected. The ocular abnormalities of mutation-positive patients with Myhre syndrome in the literature are summarized in Table 1. Axenfeld Rieger anomaly is characterized by an anteriorly displaced prominent Schwalbe line (posterior embryotoxon), usually with attached iris strands in combination with varying degrees of iris malformation such as iris hypoplasia, polycoria, corectopia, and ectropion uveae. Approximately half of the patients with this anomaly may suffer secondary glaucoma due to im- paired, abnormal aqueous outflow. Glaucoma may be present at birth or may not occur until adulthood [Idrees et al., 2006]. Furthermore, corectopia, which is a possible manifestation of the Axenfeld Rieger anomaly, is generally associated with lens dislocation and has been described in many genetic disorders, including connective tissue disorders or other malformation syndromes, that have different molecular etiologies [Colley et al., 1991; Alagia et al., 2018]. In the present report, our patient has been under medication for glaucoma since the age of 8; therefore, we suggest that patients with Myhre syndrome should be routinely examined by an ophthalmologist.

Hearing loss is observed in most of the patients with Myhre syndrome, but the type of hearing loss was not clearly defined in most of the cases reported. It is predominantly conductive or mixed, bilateral, and of any degree of severity. It also can be sensorineural. The underlying etiology of the hearing loss is often unclear or unknown [Le Goff et al., 2014; Starr et al., 2017]. Ear imaging techniques were rarely available in the reported cases but could display congenital malformations or other abnormalities in the middle or inner ear [Le Goff et al., 2014]. Table 2 describes the middle and inner ear findings of the mutation-positive patients with Myhre syndrome in the literature. EVA is the most common radiological abnormality in children with hearing loss, but the severity of the hearing impairment is variable. However, the patients may have severe or profound hearing loss that necessitates cochlear implantation [Archibald et al., 2019]. The association between hearing loss and EVA is described in syndromic and nonsyndromic disorders [Aimoni et al., 2017]. To our knowledge, the present case is the first report of bilateral EVA in a patient with Myhre syndrome, 
and this malformation may be one of the causative factors for hearing loss in this syndrome. Consequently, we suggest that temporal bone CT should be performed to evaluate the structural malformations of the inner ear, especially in patients with hearing loss.

Cardiovascular anomalies have been reported in approximately $70 \%$ of the patients. The most common congenital heart defects are large patent ductus arteriosus, atrial and ventricular septal defects as well as stenosis of aortic and mitral valves [Lin et al., 2016]. Additionally, peripheral vascular stenosis, pericardial disease, and cardiomyopathy can also be present. Patients may also exhibit several long-term complications including obesity, arterial hypertension, bronchopulmonary insufficiency, laryngotracheal stenosis, pericarditis, and recurrent infections [Le Goff et al., 2014]. In the present case, left ventricular hypertrophy was detected, and the patient suffered from arterial hypertension. Consequently, especially cardiac and respiratory follow-up should be offered to these patients for the management of these complications.

\section{Conclusion}

We reported a child with Myhre syndrome due to a SMAD4 mutation who had bilateral Axenfield Rieger anomaly with secondary glaucoma and bilateral EVA. Visual and hearing problems are common clinical features in the patients, and we suggest that these features will contribute to a better understanding and knowledge of the clinical spectrum of Myhre syndrome.

\section{Acknowledgment}

We are grateful to the family for participating in this study.

\section{Statement of Ethics}

Written informed consent was obtained from the parents, and investigations were conducted in line with the principles detailed by the Declaration of Helsinki.

\section{Disclosure Statement}

The authors have no conflicts of interest to disclose.

\section{References}

-Aimoni C, Ciorba A, Cerritelli L, Ceruti S, Caputo V, Bocchinfuso G, Castori M, Traversa A, Skarżyński PH, Hatzopoulos S: Enlarged vestibular aqueduct: audiological and genetical features in children and adolescents. Int J Pediatr Otorhinolaryngol 101:254-258 (2017).

-Alagia M, Cappuccio G, Pinelli M, Torella A, Brunetti-Pierri R, et al: A child with Myhre syndrome presenting with corectopia and tetralogy of Fallot. Am J Med Genet A 176:426-430 (2018).

-Alape D, Singh R, Folch E, Fernandez Bussy S, Agnew A, Majid A: Life-threatening multilevel airway stenosis due to Myhre syndrome. Am J Respir Crit Care Med (2019), E-pub ahead of print.

- Archibald HD, Ascha M, Gupta A, Megerian C, Otteson T: Hearing loss in unilateral and bilateral enlarged vestibular aqueduct syndrome. Int J Pediatr Otorhinolaryngol 118: 147-151 (2019).

Artemios P, Areti S, Katerina P, Helen F, Eirini T, Charalambos P: Autism spectrum disorder and psychiatric comorbidity in a patient with Myhre syndrome. J Autism Dev Disord 49: 3031-3035 (2019).

- Burglen L, Héron D, Moerman A, Dieux-Coeslier A, Bourguignon JP, et al: Myhre syndrome: new reports, review, and differential diagnosis. J Med Genet 40:546-551 (2003). Pizzuti A, et al: Novel SMAD4 mutation causing Myhre syndrome. Am J Med Genet A 164A:1835-1840 (2014).

Colley A, Lloyd IC, Ridgway A, Donnai D: Ectopia lentis et pupillae: the genetic aspects and differential diagnosis. J Med Genet 28:791-794 (1991).

Erdem HB, Sahin I, Tatar A: Myhre syndrome with novel findings: bilateral congenital cortical cataract, bilateral papilledema, accessory nipple, and adenoid hypertrophy. Clin Dysmorphol 27:12-14 (2018).

Garavelli L, Maini I, Baccilieri F, Ivanovski I, Pollazzon $M$, et al: Natural history and lifethreatening complications in Myhre syndrome and review of the literature. Eur J Pediatr 175:1307-1315 (2016).

Idrees F, Vaideanu D, Fraser SG, Sowden JC, Khaw PT: A review of anterior segment dysgeneses. Surv Ophthalmol 51:213-231 (2006).

Kenis C, Verstreken M, Gieraerts K, De Foer B, Van der Aa N, et al: Bilateral otospongiosis and a unilateral vestibular schwannoma in a patient with Myhre syndrome. Otol Neurotol 35:e253-e255 (2014).

Le Goff C, Michot C, Cormier-Daire V: Myhre syndrome. Clin Genet 85:503-513 (2014).

Li J, Qin Y, Zhao FK, Wu D, He XF, et al: Anterior segment dysgenesis correlation with epithelial-mesenchymal transition in Smad4 knockout mice. Int J Ophthalmol 9:943-947 (2016).
Lin AE, Michot C, Cormier-Daire V, L'Ecuyer TJ, Matherne GP, et al: Gain-of-function mutations in SMAD4 cause a distinctive repertoire of cardiovascular phenotypes in patients with Myhre syndrome. Am J Med Genet A 170: 2617-2631 (2016).

Michot C, Le Goff C, Mahaut C, Afenjar A, Brooks AS, et al: Myhre and LAPS syndromes: clinical and molecular review of 32 patients. Eur J Hum Genet 22:1272-1277 (2014).

Myhre SA, Ruvalcaba RH, Graham CB: A new growth deficiency syndrome. Clin Genet 20: 1-5 (1981)

Nomura R, Miyai K, Nishimura G, Kashimada K, Morio T: Myhre syndrome: age-dependent progressive phenotype. Pediatr Int 59:12051206 (2017).

Starr LJ, Lindor NM, Lin AE: Myhre Syndrome, in Adam MP, Ardinger HH, Pagon RA, et al (eds): GeneReviews ${ }^{\circledR}$ [Internet] (University of Washington, Seattle 1993-2019). Initial posting: April 13, 2017.

Whiteford ML, Doig WB, Raine PA, Hollman AS, Tolmie JL: A new case of Myhre syndrome. Clin Dysmorphol 10:135-140 (2001).

Yu KP, Luk HM, Chung BH, Lo IF: Myhre syndrome: a report of six Chinese patients and literature review. Clin Dysmorphol 28:145150 (2019). 